\title{
Computing Viable Sets and Reachable Sets to Design Feedback Linearizing Control Laws Under Saturation
}

\author{
Meeko Oishi, Ian Mitchell, Claire Tomlin, Patrick Saint-Pierre
}

\begin{abstract}
We consider feedback linearizable systems subject to bounded control input and nonlinear state constraints. In a single computation, we synthesize 1) parameterized nonlinear controllers based on feedback linearization, and 2) the set of states over which this controller is valid. This is accomplished through a reachability calculation, in which the state is extended to incorporate input parameters. While we use a HamiltonJacobi formulation, a viability approach is also feasible. The result provides a mathematical guarantee that for all states within the computed set, there exists a control law that will simultaneously satisfy two separate goals: envelope protection (no violation of state constraints), and stabilization despite saturation. We apply this technique to two real-world systems: the longitudinal dynamics of a civil jet aircraft, and a twoaircraft, planar collision avoidance scenario. The result, in both cases, is a feasible range of input parameters for the nonlinear control law, and a corresponding controlled invariant set.
\end{abstract}

\section{INTRODUCTION}

Aircraft flight management systems blend a pilot's or autopilot's control input with a variety of state and input constraints, in order to achieve goals such as management of control saturation, trajectory generation and tracking, and envelope protection. In complex systems such as aircraft, many of these control goals must be achieved simultaneously. Blindly implementing control schemes for multiple goals (e.g. envelope protection and stabilization) can result in chattering, instability, and other counterintuitive phenomenon, when the controller switches between multiple, independently designed control laws. Existing flight management systems are designed mostly through ad-hoc rules to avoid these types of problems. While the resultant systems are

This work was supported by M. Oishi's Harry S. Truman Postdoctoral Fellowship in National Security Science and Engineering at Sandia National Laboratories (operated by Sandia Corporation, a Lockheed Martin Company, for the US DoE's NNSA under Contract DE-AC04-94-AL85000), C. Tomlin's NSF Career Award, by the Defense Advanced Research Projects Agency (DARPA) under the Software Enabled Control Program (AFRL Contract F33615-99-C-3014), and by the DoD Multidisciplinary University Research Initiative (MURI) program administered by the Office of Naval Research under Grant N00014-02-1-0720.

M. Oishi is an Assitant Professor with the Department of Electrical and Computer Engineering, Vancouver, BC, Canada moishi@ece.ubc.ca

I. Mitchell is an Assistant Professor with the Department of Computer Science, University of British Columbia, Vancouver, BC, Canada mitchellacs.ubc.ca

C. Tomlin is an Associate Professor with the Department of Aeronautics and Astronautics, Stanford University, Stanford, CA, USA, and an Associate Professor with the Department of Electrical Engineering and Computer Science, University of California, Berkeley, CA, USA tomlinastanford.edu

P. Saint-Pierre is a Professor with Universite Paris IX-Dauphine, France saint-pierredviab. dauphine.fr extensively tested through costly simulations to help identify unanticipated problems, it is physically impossible to test all possible initial conditions. Problems which go undetected through this design and simulation process can result in "automation surprises" [1] and other problematic behaviors in actual aircraft operation.

As an example, consider an incident in Paris-Orly, France, in which the pilot unknowingly activated envelope protection control laws during the aircraft's final approach to landing. Upon reaching a speed excessive for the aircraft's aerodynamic configuration, the autopilot initiated an altitudechange maneuver, in order to avoid structural damage to the wing. This initially caused the aircraft to climb steeply. The autothrottles increased thrust, and the pilots attempted to use the aircraft's control surfaces to make the aircraft descend. However, the envelope protection control laws overrode the pilot's actions, commanding a high angle of attack, and eventually reaching a stall. The flight crew managed to regain control of the aircraft, then successfully completed a manual landing [2], [3].

New methods and tools in reachability analysis can provide an alternative framework for control design in safetycritical systems such as civil jet aircraft. These methods provide a mathematical guarantee of the modeled system's behavior, in the presence of state and input constraints. Physical constraints arising from aerodynamic envelope protection, such as the speed at which an aircraft can stall, can be incorporated as constraints on the continuous statespace. Actuator saturation can be incorporated as an input constraint resulting in bounded control authority. When other goals, such as trajectory tracking, are incorporated into this analysis, multiple objectives can be simultaneously served through a single computational synthesis.

We show how to synthesize, in a single computation, controllers which simultaneously satisfy two separate objectives: 1) envelope protection, and 2) stabilization under input saturation.

To address the problem of envelope protection, we define safety as the ability to remain within a set of constraints in the continuous state-space, despite bounded control authority. We seek to determine the controlled invariant set: the subset of safe states in which we can guarantee the state of the system can always remain. We find this set by computing its converse: the set of states which are themselves unsafe or which give rise to trajectories which become unsafe [4]. This converse is simply the backwards reachable set of the unsafe states, and we can compute backwards reachable 
sets using existing techniques based on Hamilton-Jacobi partial differential equations (HJ PDEs). In addition to the backwards reachable set, these techniques also provide setvalued control laws, so by taking set complements we can find both the controlled invariant set and the control signals which make it invariant. We draw on the HamiltonJacobi techniques here because of their sub-grid accuracy and success in previous aircraft applications [5], [6], [7], however viability techniques could also be used. Viability theory [8] and numerical algorithms [9] have been developed to compute viability kernels and capture basins for continuous systems, and also extended to hybrid systems [10], [11]. These are computationally based on a minimum-timeto-reach formulation [12].

To address stabilization under saturation, we parameterize feedback linearizing control laws subject to bounded control input, such that the parameters reflect system performance goals (e.g. damping, overshoot). We formulate constraints that input saturation and stability place on the input parameters. Feedback linearization is a popular technique for differentially flat systems [13], [14], but can generate inputs with high-magnitude. Synthesizing non-saturating feedback linearizing control laws is a non-trivial problem [15], [16], [17] for stabilization [18] as well as for tracking [19]. Trajectory generation for differentially flat systems often involves saturation and rate constraints [20], [21]. Other common techniques to incorporate state and input constraints are model predictive control [22], [23], [24], and control Lyapunov functions [25], [26], however finding such functions is often difficult and done heuristically. For linear systems, quadratic Lyapunov functions can be synthesized [27], [28]. A variety of techniques have been investigated to control linear systems with constraints [29], [30], [31], [32].

Our method is novel in its use of reachability analysis to perform multiobjective controller synthesis which assures stabilization, envelope protection, and input non-saturation. While a standard reachability analysis guarantees envelope protection and input non-saturation, the resultant control law would not guarantee stability and may not be implementable - it could be bang-bang or cause chattering. Therefore, our approach uses a reachability calculation with additional constraints to ensure that the resultant control law meets all the desired objectives and is implementable, as well. By extending the state to include the input parameters, we can include constraints for stability, saturation, and envelope protection in a single reachability analysis. Hence in a single step, we find non-saturating feedback linearizing controllers through application of reachability techniques which guarantee envelope protection.

The paper is organized as follows: We first formulate the problem we wish to solve and provide a brief description of the reachability computation and the resultant invariant set. We demonstrate this method on three examples: a double integrator, a nonlinear model of a two-input, twostate system representing the longitudinal dynamics of a civil jet aircraft, and a two-aircraft, three-dimensional cooperative collision avoidance model. For each example, we design a parameterized feedback linearizing controller designed to stabilize despite input saturation. We formulate the initial cost function for an extended-state reachability computation, and discuss the computed results: 1) controllers which are guaranteed to stabilize the system without violating state constraints or saturating the input, and 2) the set of states from which any given controller can be applied. Our current efforts involve synthesis of switched controllers by choosing from a set of the computed controllers to minimize the time to reach the origin. We end with conclusions and directions for future work.

\section{Problem Formulation}

We begin with the simplest case. Consider the input-output full-state feedback linearizable system

$$
\begin{aligned}
\dot{x} & =f(x)+g(x) u, x \in \mathcal{X} \subseteq \mathbb{R}^{n}, u \in \mathbb{R} \\
y & =h(x), y \in \mathbb{R}
\end{aligned}
$$

with bounded input $u \in \mathcal{U}$, and constraint set $\mathcal{C} \subset \mathbb{R}^{n}$ which encodes the set of states which satisfy the constraints on the system (e.g., speeds above the aircraft's stall speed). We express the state constraints through the inequality

$$
\mathcal{C}=\{x \mid c(x) \geq 0\} .
$$

The feedback linearizing control law to stabilize (1) around the equilibrium $x^{*}=0$ is

$$
u(x, \beta)=\frac{1}{L_{g} h}\left(-L_{f}^{n-1} h-\sum_{i=0}^{n-1} \beta_{i} x^{(i)}\right)
$$

with Lie derivatives $L_{f} h=\frac{\partial h}{\partial x} f(x), L_{f}^{2} h=\frac{\partial}{\partial x}\left(L_{f} h\right), \cdots$, $L_{f}^{n-1} h=\frac{\partial}{\partial x}\left(L_{f}^{n-2} h\right)$, and $x^{(i)}$ the $i$ th time derivative of $x$. Constant coefficients $\beta=\left[\beta_{0}, \beta_{1}, \cdots, \beta_{n-1}\right]$ are chosen such that the polynomial in $s$

$$
s^{n}+\sum_{i=0}^{n-1} \beta_{i} s^{i}
$$

is Hurwitz. With this control law, the resultant closed-loop system will be linear and stable. However, in order to prevent saturation, the control $u(x, \beta)$ must remain within its allowable bounds $\mathcal{U}$ for a constant $\beta$ for $x \in \mathcal{X}$.

$$
u_{\min } \leq u(x, \beta) \leq u_{\max }
$$

Define the reachable set as the set of states in $\mathcal{C}$ for which all values of a measurable function $u(\cdot)$ in $\mathcal{U}$ drive the system state out of the constraint set $\mathcal{C}$. We presume that the equilibrium $x^{*}=0 \in \mathcal{C}$ is contained in the constraint set. We compute the reachable set and its complement, known as the invariant set, through Hamilton-Jacobi techniques.

We wish to satisfy two goals with a single controller: 1) envelope protection, and 2) stabilization under saturation. Further, we wish to determine the largest set of states from which this controller is guaranteed to fulfill these goals.

Statement of Problem 1: Given the dynamical system (1), with state constraints (2), and with a feedback linearizing control law $u(x, \beta)$ (3) parameterized by a constant vector $\beta \in \mathbb{R}^{n}$, determine 1) the invariant set $\mathcal{W}$, which is the 
largest set of states $x$ for a given non-saturating controller $u(x, \beta)$ that will reach the origin without violating the state constraints $x \in \mathcal{C}$, 2) $\beta$ such that the feedback linearizing control law is both non-saturating (5) and stable (4).

\section{METHOD}

We first append the parameter vector $\beta$ to the state such that $\tilde{x}=[x, \beta] \in \mathbb{R}^{2 n}$. The extended dynamics

$$
\begin{aligned}
& \dot{x}=f(x)+g(x)\left(-L_{f}^{n-1} h-\frac{1}{L_{g} h} \sum_{i=0}^{n-1} \beta_{i} x^{(i)}\right) \\
& \dot{\beta}=0
\end{aligned}
$$

ensure that $\beta$ remains a constant in the reachability computation. For first and second-order systems, the requirements for stability (4) simplify to

$$
\beta_{i}>0
$$

while for higher-order systems, (4) can be represented as a set of inequalities in $\beta_{i}$.

Through reachability analysis and controller synthesis we can determine the backwards reachable set $\overline{\mathcal{W}(t)}$ and its complement, the controlled invariant set $\mathcal{W}(t)$. Given a dynamically evolving system (6) and a constraint set $\tilde{\mathcal{C}}$, we define the backwards reachable set as the set of all states which will exit the constraint set $\tilde{\mathcal{C}}$ in the time $[0, t]$. The controlled invariant set is simply the complement of this result. To calculate the backwards reachable set, define a continuous function $J_{0}: \mathcal{X} \times \mathbb{R}^{n} \rightarrow \mathbb{R}$ such that

$$
\tilde{\mathcal{C}}=\left\{x \in \mathcal{X}, \beta \in \mathbb{R}^{n} \mid J_{0}(x, \beta) \geq 0\right\} .
$$

The backwards reachable set $\overline{\mathcal{W}(t)}$ can be found by solving the terminal value Hamilton-Jacobi (HJ) partial differential equation (PDE) [33], [4], [6]

$$
\begin{aligned}
\frac{\partial J(\tilde{x}, t)}{\partial t}+\min \left[0, H\left(\tilde{x}, \frac{\partial J(\tilde{x}, t)}{\partial \tilde{x}}\right)\right] & =0 \quad \text { for } t<0 ; \\
J(\tilde{x}, 0) & =J_{0}(\tilde{x}) \text { for } t=0 ;
\end{aligned}
$$

As shown in [6], the implicit representation of the backwards reachable set is $\overline{\mathcal{W}(t)}=\left\{x \in \mathcal{X}, \beta \in \mathbb{R}^{n} \mid J(\tilde{x},-t) \leq 0\right\}$. If (9) converges as $t \rightarrow-\infty$, then $J(\tilde{x},-t) \rightarrow J(\tilde{x})$ and the reachable set converges to a fixed point $\overline{\mathcal{W}(t)} \rightarrow \overline{\mathcal{W}}$.

In order to solve Problem 1, we incorporate the state bounds, non-saturation constraints, and stability constraints into the initial cost function

$$
\begin{array}{r}
J_{0}(x, \beta)=\min \left\{J_{0}^{\text {state }}(x, \beta), J_{0}^{\text {sat }-\max }(x, \beta),\right. \\
\left.J_{0}^{\text {sat }- \text { min }}(x, \beta), J_{0}^{\text {stability }}(x, \beta)\right\}
\end{array}
$$

for which we define the functions

$$
\begin{aligned}
J_{0}^{\text {state }}(x, \beta) & =c(x) \\
J_{0}^{\text {sat }- \text { max }}(x, \beta) & =u_{\max }-u(x, \beta) \\
J_{0}^{\text {sat }- \text { min }}(x, \beta) & =u(x, \beta)-u_{\text {min }} \\
J_{0}^{\text {stability }}(x, \beta) & =\beta_{i}
\end{aligned}
$$

such that they are positive in those regions where the constraints are satisfied. We then define the Hamiltonian as

$$
\begin{aligned}
H\left(\tilde{x}, \frac{\partial J}{\partial \tilde{x}}\right)= & \left(\frac{\partial J}{\partial x}\right)^{T}\left(f(x)+g(x)\left(-L_{f}^{n-1} h\right.\right. \\
& \left.\left.-\frac{1}{L_{g} h} \sum_{i=0}^{n-1} \beta_{i} x^{(i)}\right)\right)+\left(\frac{\partial J}{\partial \beta}\right)^{T} \cdot 0,
\end{aligned}
$$

as opposed to the standard formulation in which

$$
H\left(x, \frac{\partial J}{\partial x}\right)=\max _{u \in \mathcal{U}}\left(\frac{\partial J}{\partial x}^{T}(f(x)+g(x) u)\right) .
$$

The control law which results from (13) will provide envelope protection and prevent input saturation, however it may be bang-bang, and because it is a measurable function, may not be implementable. However, by construction, the control law used in (12) will be smooth, stabilize (1), ensure envelope protection, and will not saturate. The invariant set computed using (13) will contain the invariant set computed using (12).

By appending $\beta$ to the state and prescribing zero dynamics to it, we have assured that $\beta$ is constant. This is required to guarantee stability. The result of the reachability computation is the largest set of states $x$ for a given input parameter $\beta$ for which trajectories that begin in this set and are controlled through (3) will reach the origin without violating any state constraints (2) or saturating the input (5).

Remark: We have solved Problem 1 through the above formulation of constraints into the reachability calculation with initial conditions (10),(11) and Hamilton-Jacobi PDE (12). The feedback linearizable system (1) with control input (3) parameterized by a constant $\beta$ which satisfies (5) and (4), will result in a system locally stable around $x=0$. This control law will not saturate for states in the invariant set $x \in \mathcal{W}$.

The advantage of this framework is that the computed result inherently meets the required constraints for both stability and non-saturation, while maintaining invariance within the constraint set. For a given value of $\beta$, the computed result in $x$ is the region of the state-space for which the specified controller will stabilize, will not saturate, and will not violate any of the state bounds. As there are generally no analytic ways to synthesize such $\beta$, this computation provides an alternative to simply picking various $\beta$ and checking whether they fulfill the conditions for stability and nonsaturation.

While we have presented our method for the simplest case, a SISO system with full-state feedback, this method also generalizes to MIMO systems which are full-state feedback linearizable or which can be transformed into full-state feedback linearizable form.

At first glance, solving (9), (12) involves a reachability calculation in $2 n$ dimensions - no small feat due to the complexity of the calculation, $\mathcal{O}\left(d^{2 n}\right)$ with $d$ grid points in $2 n$ dimensions. However, by exploiting structure in the pole placement, we can reduce the computation to $\mathcal{O}\left(d^{n+1}\right)$, with $d$ grid points in $n+1$ dimensions. Consider a simple double integrator, $\beta \in \mathbb{R}_{+}^{2}$. If we collocate poles on the real line with $\beta_{1}=\eta^{2}, \beta_{2}=2 \eta$, the computation is reduced from 4 to 3 


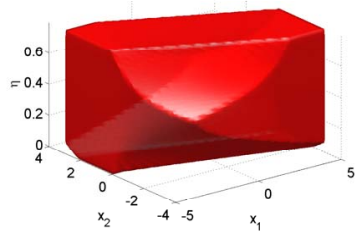

Fig. 1. Invariant set $\mathcal{W}_{\beta}$ plotted in $\left[x_{1}, x_{2}, \eta\right]$ for $\eta=0.35$ corresponding to two real poles at -0.35 .

dimensions. In addition, because $\dot{\beta}$ does not depend on $x$, the reachable set can be computed for various $\beta$ independently, e.g. on parallel computers to sweep through $\beta$-space. This parallelization is generally not possible for reachable sets with coupled dynamics.

\section{EXAMPLES}

\section{A. Double Integrator}

To demonstrate this method, consider the system $\ddot{x}=u$, with state $x=\left[x_{1}, x_{2}\right] \in \mathcal{C}=\mathcal{X}=\left[\underline{x}_{1}, \bar{x}_{1}\right] \times\left[\underline{x}_{2}, \bar{x}_{2}\right]$, input $u \in \mathcal{U}=\left[u_{\min }, u_{\max }\right]$, and output $h=x_{2}$. We design a feedback linearizing control law, $u(x, \beta)=-\beta_{1} x_{1}-\beta_{2} x_{2}$, with $\beta_{1}, \beta_{2} \in \mathbb{R}^{+}$, such that the resultant closed-loop system is stable.

$$
\dot{x}=\left[\begin{array}{cc}
0 & 1 \\
-\beta_{1} & -\beta_{2}
\end{array}\right] x
$$

As state dimensionality is at a premium in the reachability calculation, we consider two cases: 1) two real poles when $\beta_{1}=\eta^{2}, \beta_{2}=\eta$, and 2) two complex poles when $\beta_{1}=$ $\beta_{2}=\eta$. For these two cases, the reachability computation will proceed in 3 , as opposed to 4 , dimensions. The constraint set $\mathcal{C} \subseteq \mathcal{X} \times \mathbb{R}^{+}$incorporates both the state constraint $x \in \mathcal{C}$ as well as the input constraint $u(x, \beta) \in \mathcal{U}$.

The saturation constraints (5), state constraints, and stability constraints are formulated as

$$
\begin{aligned}
& J_{0}^{\mathrm{sat}}(x, \beta)=\min \left\{u_{\max }-\beta_{1} x_{1}-\beta_{2} x_{2},\right. \\
& \left.-\beta_{1} x_{1}-\beta_{2} x_{2}-u_{\min }\right\} \\
& J_{0}^{\text {state }}(x)=\min \left\{\bar{x}_{1}-x_{1}, x_{1}-\underline{x}_{1}, \bar{x}_{2}-x_{2}, x_{2}-\underline{x}_{2}\right\} \\
& J_{0}^{\text {stability }}(\beta)=\eta \text {. }
\end{aligned}
$$

For the reachability computation, we combine the above three functions into one initial cost function

$$
J_{0}(x, \beta)=\min \left\{J_{0}^{\text {sat }}(x, \beta), J_{0}^{\text {state }}(x), J_{0}^{\text {stability }}(\beta)\right\}
$$

Each horizontal slice in Figures 1 and 2 represents the invariant set in $\left[x_{1}, x_{2}\right]$ for a given control parameter $\eta$. It is the set of initial conditions for which the state will be driven to the equilibrium without saturating the input or violating the state constraints. While for this trivial system, non-saturating, stabilizing controllers can be calculated by hand, for general nonlinear systems, this is not possible. Figures 3 and 4 show the largest invariant sets, maximized over $\beta$ through a simple post-processing calculation. By contrast, while the controlled invariant set calculated for

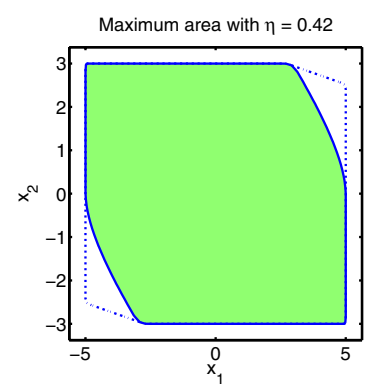

Fig. 3. Largest invariant set with two real poles, plotted in $\left(x_{1}, x_{2}\right)$ for $\eta=0.42$. The region contained the dotted lines is the initial constraint set for which $J_{0}(x, \beta) \geq 0$.

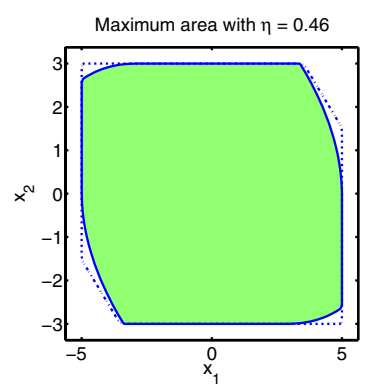

Fig. 4. Largest invariant set with an imaginary pair of poles, plotted in $\left(x_{1}, x_{2}\right)$ for $\eta=0.46$. The region contained by the dotted lines is the initial constraint set for which $J_{0}(x, \beta) \geq 0$.

$\ddot{x}=u$, measurable $u \in \mathcal{U}$ (without a feedback linearizing control law specified) will contain both of these invariant sets, the input signal required may not be implementable and will not stabilize the system.

\section{B. Aerodynamic Envelope Protection}

We model the longitudinal dynamics of a conventional aircraft as a nonlinear system

$$
\begin{aligned}
m \dot{V} & =T-D(\alpha, V)-m g \sin \gamma \\
m V \dot{\gamma} & =L(\alpha, V)-m g \cos \gamma
\end{aligned}
$$

with state $x=[V, \gamma] \in \mathcal{X}=\mathbb{R}^{+} \times \mathbb{R}$ (corresponding to speed $V$ and flight path angle $\gamma$ ) and input $u=[T, \alpha] \in$ $\mathcal{U}=\left[T_{\min }, T_{\max }\right] \times\left[\alpha_{\min }, \alpha_{\max }\right]$ (corresponding to thrust $T$ and angle of attack $\alpha$ ). The state constraints are given by $x \in \mathcal{C}=\left[V_{\min }, V_{\max }\right] \times\left[\gamma_{\min }, \gamma_{\max }\right] \subseteq \mathcal{X}$. Physical constants are mass $m=200000 \mathrm{~kg}$, gravitational constant $g=9.81 \mathrm{~m} / \mathrm{s}^{2}$, and the sets $\mathcal{X}=[63.79,97.74] \times[-6,6], \mathcal{U}=$ $[0,686700] \times[-5,17]$ are determined by standard aircraft operating conditions. Drag and lift are given by

$$
\begin{aligned}
D(\alpha, V) & =V^{2}\left(a+b c_{L}(\alpha)^{2}\right) \\
L(\alpha, V) & =c V^{2} c_{L}(\alpha)
\end{aligned}
$$

with coefficient of lift $c_{L}(\alpha)=c_{L_{0}}+c_{L_{\alpha}} \alpha$, and positive constants $a=6.5106, b=12.6585, c=262.0275, c_{L_{0}}=$ 0.4225 , and $c_{L_{\alpha}}=5.105$ as in the Flaps-20 configuration of the large civil jet aircraft with its landing gear up [5]. The aircraft should track a reference speed $V_{r}=90 \mathrm{~m} / \mathrm{s}$ and a reference flight path angle $\gamma_{r}=0^{\circ}$.

We rewrite (17) in feedback linearizable form as

$\left[\begin{array}{c}m \dot{V} \\ m V \dot{\gamma}\end{array}\right]=\left[\begin{array}{c}-m g \sin \gamma-a V^{2} \\ -m g \cos \gamma\end{array}\right]+\left[\begin{array}{cc}1 & 0 \\ 0 & c V^{2}\end{array}\right]\left[\begin{array}{c}\bar{u}_{1} \\ \bar{u}_{2} \\ (19)\end{array}\right.$

by defining a new input through an invertible transformation

$$
\bar{u} \triangleq \phi(u)=\left[\begin{array}{c}
-b V^{2} c_{L}(\alpha)^{2}+T \\
c_{L}(\alpha)
\end{array}\right] .
$$

With outputs $y=[V, \gamma]$, equation (19) has relative degree 1 for each output. In order to track a desired reference state $x_{r}=\left[V_{r}, \gamma_{r}\right]$, define an auxiliary input $v=\left[-\beta_{1}(V-\right.$ 

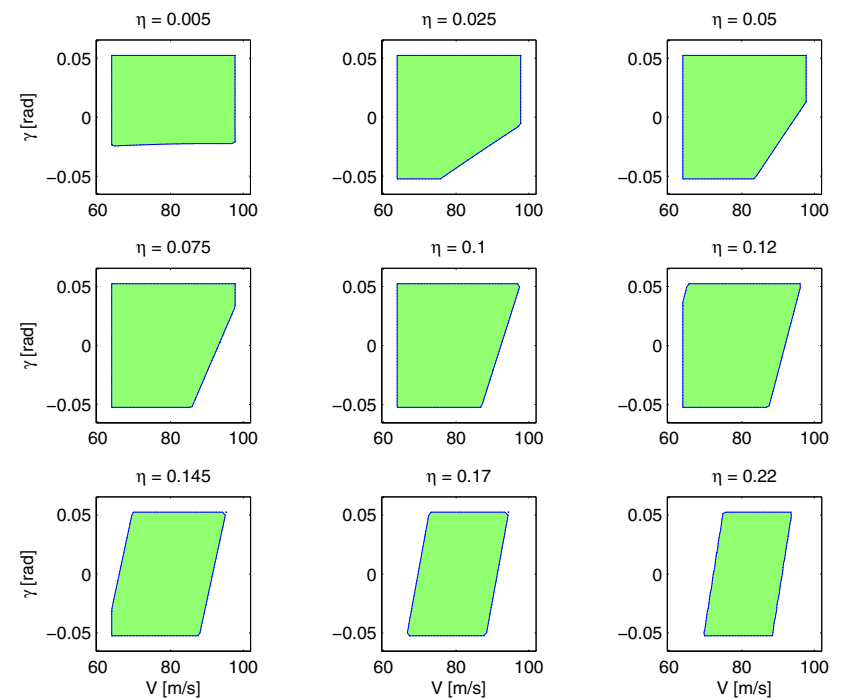

Fig. 5. Horizontal slices of Figure 6 show the invariant set in $(V, \gamma)$ at various $\eta$ which correspond to stabilizing and non-saturating control laws.

$\left.\left.V_{r}\right),-\beta_{2}\left(\gamma-\gamma_{r}\right)\right]$, with $\beta_{1}, \beta_{2} \in \mathbb{R}^{+}$. The control law

$$
u(x, \beta)=\phi^{-1}\left(\left[\begin{array}{c}
m g \sin \gamma+a V^{2}-m \beta_{1}\left(V-V_{r}\right) \\
\frac{1}{c V^{2}}\left(m g \cos \gamma-m V \beta_{2}\left(\gamma-\gamma_{r}\right)\right)
\end{array}\right]\right)_{(21)}
$$

results in linear dynamics, stable around $x_{r}$.

Assuming that $\beta_{1}=\beta_{2}=\eta$, we incorporate the state constraints, input constraints, and stability constraints into the initial cost function:

$$
J_{0}(x, \beta)=\min \left\{J_{0}^{\mathrm{sat}}(x, \beta), J_{0}^{\mathrm{env}}(x), \beta\right\}
$$

with $J_{0}^{\mathrm{sat}}(x, \beta)=\min \left\{u_{\max }-u(x, \beta), u(x, \beta)-u_{\min }\right\}$, $J_{0}^{\text {state }}(x, \beta)=\min \left\{V_{\max }-V, V-V_{\min }, \gamma_{\max }-\gamma, \gamma-\gamma_{\min }\right\}$.

The result of the reachability calculation is shown in Figure 6 for combinations of $[V, \gamma, \eta]$. For clarity, crosssections of $[V, \gamma]$ for various $\eta$ are shown in Figure 5. As the control parameter increases, less of the aerodynamic flight envelope $(V, \gamma)$ is controllable, due mainly to input saturation. The uncontrollable portions of the $(V, \gamma)$ envelope at all $\eta$ in the lower right quadrant correspond to descent at high speeds - this is a well-known issue for landing aircraft, in which the aircraft is very close to a stall condition.

As with the double integrator example, we numerically evaluate the computational result to determine the control parameter $\eta$ which generates the largest invariant set. For each $\eta$, we count the number of grid points which have a positive value. For this scenario, the maximum number of positive-valued grid points occurs at $\eta=0.045$. This result could be useful for maximizing the controllable portion of the aircraft's aerodynamic envelope. The envelope with the largest area in $(V, \gamma)$-space will allow for operation in more combinations of speed and flight path angle than any other envelope in the computed set $\mathcal{W}$.

In situations in which it is imperative to have a larger controllable set in regions of high speed and high descent rate, unstable controllers might be useful. An unstable controller

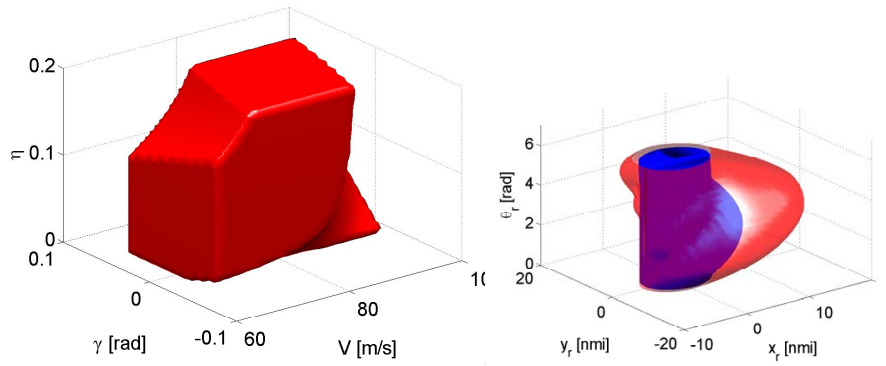

Fig. 6. Invariant set in $[V, \gamma, \eta]$. For a given $\eta$, states inside the shaded region will reach $\left(V_{r}, \gamma_{r}\right)$ without saturating the input or violating the aerodynamic envelope.

Fig. 7. Invariant set in $\left[x_{r}, y_{r}, \theta_{r}\right]$ for $\beta=1.10 \times 10^{-3}$ for the collision avoidance scenario.

in this region of the aerodynamic flight envelope would need to be combined with stabilizing controllers in other regions of the aerodynamic flight envelope in a carefully defined switching scheme. While the practicalities of aircraft certification and pilot training would likely prevent unstable controllers from implementation onboard civil jet aircraft, other platforms which require agility in all areas of the aerodynamic envelope (such as fighter aircraft or helicopters) might benefit from such a strategy.

\section{Cooperative Collision Avoidance}

We model the relative dynamics of two aircraft traveling at a constant speed $V=0.125 \mathrm{nmi} / \mathrm{s}$,

$$
\left[\begin{array}{c}
\dot{x}_{r} \\
\dot{y}_{r} \\
\dot{\theta}_{r}
\end{array}\right]=V\left[\begin{array}{c}
\cos \left(\theta_{r}-\theta_{R}\right)-1 \\
\sin \left(\theta_{r}-\theta_{R}\right) \\
0
\end{array}\right]-\frac{V}{g}\left[\begin{array}{rr}
y_{r} & 0 \\
-x_{r} & 0 \\
-1 & 1
\end{array}\right] u
$$

with relative position $x_{r}, y_{r}$, relative heading $\theta_{r}$, turn rates $u_{1}, u_{2} \in[-\tan (2 \pi / 9), \tan (2 \pi / 9)]$, and constant $g=5.3 \times$ $10^{-3} \mathrm{nmi} / \mathrm{s}^{2}$. As opposed to the competitive model presented in [34], we assume cooperative, centralized control over both aircraft. For ease of notation, we write (23) as $\dot{x}=F(x)+$ $G(x) u$, with $F(x) \in \mathbb{R}^{3}$ and $G(x) \in \mathbb{R}^{3 \times 2}$. The aircraft must remain at least $R=5 \mathrm{nmi}$ apart at all times, so the state is constrained by

$$
J_{0}^{\text {radius }}(x)=x_{r}^{2}+y_{r}^{2}-R^{2} \geq 0
$$

Assuming cooperation between the two aircraft and full-state output $y=\left[x_{r}, y_{r}, \theta_{r}\right]$, the feedback linearizing control

$$
u(x, \beta)=\left(G^{T} G\right)^{-1} G^{T}\left(\left[\begin{array}{c}
\beta_{1} x_{r} \\
\beta_{2} y_{r} \\
\beta_{3}\left(\theta_{r}-\theta_{R}\right)
\end{array}\right]-F(x)\right)
$$

tracks a desired relative heading $\theta_{R}$, subject to saturation constraints $J_{0}^{\max }(x, \beta)=u_{\max }-u(x, \beta), J_{0}^{\min }(x, \beta)=$ $u(x, \beta)-u_{\max }$. The resultant dynamics are

$$
\dot{x}=\frac{p(x) V}{x_{r}^{2}+y_{r}^{2}}\left[\begin{array}{c}
y_{r} \\
-x_{r} \\
0
\end{array}\right]+\left[\begin{array}{c}
V\left(\cos \left(\theta_{r}-\theta_{R}\right)-1\right) \\
V \sin \left(\theta_{r}-\theta_{R}\right) \\
-\beta_{3}\left(\theta-\theta_{R}\right)
\end{array}\right]
$$


with $p(x)=-y_{r}\left(\cos \left(\theta_{r}-\theta_{R}\right)-1\right)+x_{r} \sin \left(\theta_{r}-\theta_{R}\right)$. We parameterize $\beta$ by $\beta_{1}=\beta_{2}=0.1 \beta_{3}=\eta$, resulting in the additional constraint $J_{0}^{\eta}=\eta$.

The initial cost function for the reachability calculation is

$$
J_{0}(x, \beta)=\min \left\{J_{0}^{\text {radius }}(x), J_{0}^{\max }(x, \beta), J_{0}^{\min }(x, \beta), J_{0}^{\eta}(\beta)\right\}
$$

Figure 7 shows the the resultant reachable set for $\eta=$ $1.10 \times 10^{-3}$. As opposed to the two previous examples, the safe region lies outside of the shaded region. For clarity, the invariant set calculated with dynamics (23) and initial cost function (24) is also displayed in Figure 7, and contains the invariant set calculated with the prescribed controller (26) and initial cost function (27).

\section{Switched Control Laws}

We are currently exploring synthesis of stable, switched control laws that will not saturate and will not violate state constraints, in order to increase the controllable range of the state-space. We present the initial results of this work.

The result of the reachability calculation demonstrated in the previous three examples is 1) a discrete set of input parameters $\beta$, and 2) the invariant sets in $x$ corresponding to a given input $u(x, \beta)$. An immediate question arises: How can we determine which $\beta$ is "best"? In the double integrator and in the aircraft example, we chose a single value for $\beta$, which corresponded to the largest invariant set. However, there is no reason to be restricted to a single value of $\beta$ : a reasonable alternative is to choose a set of $\beta$, and to optimally switch between them. This might be particularly useful in systems for which the invariant sets are drastically different for various $\beta$, since enabling switching results in a larger invariant set than any of that of the individual systems alone.

Statement of Problem 2: Find the optimal state-based switching control law required to minimize the time to reach a small-radius $\mathcal{B}_{n}$ around the desired equilibrium point, given a finite set of $p$ non-saturating feedback linearizing controllers parameterized by $\beta_{1}, \ldots, \beta_{p}$.

For this computation, while either Hamilton-Jacobi or viability techniques could be implemented, we apply viability techniques that are well-tested in switched systems to compute the minimum-time-to-reach function [12].

For an impulse dynamical system described by the differential inclusion

$$
x \in F(x(t)), F(x)=\{f(x)+\tilde{g}(x) u(x, \beta), u \in \mathcal{U}\}
$$

with no state reset map, define the target set $\mathcal{T} \subseteq \mathcal{C}$ as a subset of the constraint set $\mathcal{C}$. Consider the problem of reaching $\mathcal{T}$ without leaving $\mathcal{C}$. The viability kernel with target $\operatorname{Viab}_{F}(\mathcal{C}, \mathcal{T}) \subseteq \mathcal{C}$ is the largest set of initial conditions in $\mathcal{C}$ from which at least one trajectory governed by (28) will remain in the constraint set $\mathcal{C}$ until it reaches the target $\mathcal{T}$, which we assume here is a small-radius ball $\mathcal{B}_{n}$ around the equilibrium point.

Consider the minimum-time-to-reach value function

$$
V\left(x_{0}\right)=\inf _{x_{0} \in S_{F}\left(x_{0}\right)} T_{\mathcal{C}}^{\mathcal{T}}(x(\cdot))
$$

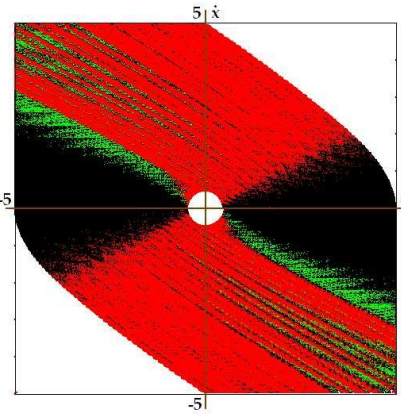

Fig. 8. In the lightest (red) regions, $\beta_{1}=[0.1225,0.70]$ is invoked. In the darkest (black) regions, $\beta_{2}=[0.30,0.30]$ is invoked; and in the medium-shade (green) regions, $\beta_{3}=[0,0]$ is invoked.

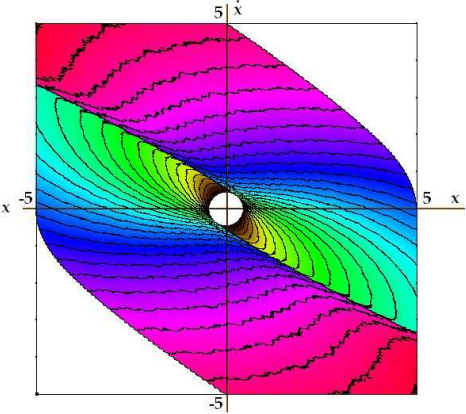

Fig. 9. Level sets of the minimum-time-to-reach computation for a switched system with dynamics corresponding to $\beta_{1}=[0.1225,0.70], \beta_{2}=$ $[0.30,0.30]$, and $\beta_{3}=[0,0]$. for initial state $x_{0}$ in the solution map $S_{F}\left(x_{0}\right)$, in which the time cost

$$
T_{\mathcal{C}}^{\mathcal{T}}(x(\cdot))=\left\{\begin{array}{l}
\inf \{t: x(t) \in \mathcal{T} \text { and } \forall s<t, x(s) \in \mathcal{C}\} \\
+\infty \text { if the constraint condition fails }
\end{array}\right.
$$

is the first time the system reaches the target when following the trajectory $x(\cdot)$. We know that the epigraph of $V$, defined as $\operatorname{Epi}(V):=\left\{(x, y) \in \mathcal{C} \times \mathbb{R}^{+}: V(x) \leq y\right\}$, coincides with a viability kernel with target associated with an extended dynamical system

$$
\operatorname{Epi}(V)=\operatorname{Viab}_{\bar{F}}\left(\mathcal{K} \times \mathbb{R}^{+}, \mathcal{T} \times \mathbb{R}^{+}\right)
$$

in which $\bar{F}(x, y)=F(x) \times\{-1\}$ and the state $x$ has been augmented to include a dimension for time [35]. The viable set is the domain of the function $V(x)$.

For the double integrator problem, we choose three parameters to represent three qualitatively different closed-loop systems (14). The parameter $\beta_{1}=[0.1225,0.7000]$ generates non-oscillatory trajectories that asymptotically approach the origin; $\beta_{2}=[0.30,0.30]$ results in damped oscillatory trajectories, and $\beta_{3}=[0,0]$ results stationary trajectories.

Figure 9 shows level sets of the minimum-time-to-reach function. The resulting control law, shown in Figure 8 demarcates regions in $\mathcal{W}$ in which the different $\beta$ should be used in order to reach $\mathcal{B}_{n}$ in minimal time.

\section{CONCLUSION}

We presented a method to determine, through a HamiltonJacobi reachability computation, the set of states in safetycritical systems which will reach the desired equilibrium without saturating the input or violating the state constraints. Thus both envelope protection and stabilization under saturation are simultaneously achieved. This involves a reachability analysis on an extended state space which incorporates a parameter from the feedback linearizing input. By incorporating the input saturation, stability, and state constraints simultaneously in the initial cost function, the resultant invariant set will be the largest set of states, given bounded input, which will stabilize the system and always 
remain within a given constraint set.

We presented two real-world examples to illustrate the method. We synthesized non-saturating feedback linearizing controllers, and determined where in the state-space these controllers could be used to guarantee not only stability and non-saturation of the input, but also invariance with respect to state constraints.

The method and three examples presented contribute to the difficult problem of determining stabilizing controllers for safety-critical systems under nonlinear state and input constraints. Many future directions of work are possible, including 1) minimization of the number of switched, nonsaturating controllers when multiple solutions to the control parameterization problem are possible, 2) alternative, less computationally exhaustive formulations to sample the parameter space $\beta$ and 3) one-step synthesis of a minimal number and optimal selection of input parameters $\beta$ for switched, non-saturating, feedback linearizing controllers.

\section{REFERENCES}

[1] E. Palmer, "Oops, it didn't arm - a case study of two automation surprises," in 8th International Symposium on Aviation Psychology, Columbus, Ohio, 1995.

[2] K. Abbott, S. Slotte, and D. Stimson, "The interfaces between flightcrews and modern flight deck systems," Federal Aviation Administration," Human Factors Team Report, June 1996.

[3] C. Billings, Aviation Automation: The Search for a Human-Centered Approach. Hillsdale, NJ: Erlbaum, 1997.

[4] C. Tomlin, J. Lygeros, and S. Sastry, "A game theoretic approach to controller design for hybrid systems," Proceedings of the IEEE, vol. 88, no. 7, pp. 949-970, 2000.

[5] M. Oishi, I. Mitchell, A. Bayen, C. Tomlin, and A. Degani, "Hybrid verification of an interface for an automatic landing," in Proceedings of the IEEE Conference on Decision and Control, Las Vegas, NV, December 2002, pp. 1607-1613.

[6] I. Mitchell, A. M. Bayen, and C. J. Tomlin, "A time-dependent Hamilton-Jacobi formulation of reachable sets for continuous dynamic games," IEEE Transactions on Automatic Control, vol. 50, no. 7, pp. 947-957, July 2005.

[7] I. Mitchell, A Toolbox of Level Set Methods, Department of Computer Science, University of British Columbia, June 2004, www.cs.ubc.ca/mitchell/ToolboxLS.

[8] J. Aubin, Viability Theory. Birkhauser, 1991.

[9] P. Saint-Pierre, "Approximation of the viability kernel," Applied Mathematics and Optimisation, vol. 29, pp. 187-209, 1994.

[10] J. Aubin, J. Lygeros, M. Quincampoix, S. Sastry, and N. Seube, "Impulse differential inclusions: a viability approach to hybrid systems," IEEE Transactions on Automatic Control, vol. 47, no. 1, pp. 2-20, 2002.

[11] P. Saint-Pierre, "Approximation of viability kernels and capture basin for hybrid systems," in European Control Conference, J. Martins de Carvalho, Ed., 2001, pp. 2776-2783.

[12] E. Cruck and P. Saint-Pierre, "Nonlinear impulse target problems under state constraint: A numerical analysis based on viability theory," SetValued Analysis, vol. 12, no. 4, pp. 383-416, December 2004.

[13] M. Fliess, J. Levin, P. Martin, and P. Rouchon, "A Lie-Backlund approch to equivalence and flatness of nonlinear systems," IEEE Transactions on Automatic Control, vol. 44, no. 5, pp. 922-937, 1999.

[14] R. Hirschorn and J. Davis, "Global output tracking for nonlinear systems," SIAM Journal of Control and Optimization, vol. 26, no. 6, pp. 1321-1330, 1988.

[15] A. Bemporad, "Reference governor for constrained nonlinear systems," IEEE Transactions on Automatic Control, vol. 43, no. 3, pp. 415-419, 1998.

[16] G. Pappas, J. Lygeros, and D. Godbole, "Stabilization and tracking of feedback linearizable systems under input constraints," in Proceedings of the IEEE Conference on Decision and Control, New Orleans, LA, December 1995, pp. 596-601.
[17] N. Kapoor and P. Daoutidis, "Stabilization of unstable systems with input constraints," in Proceedings of the American Control Conference, Seattle, WA, June 1995, pp. 3192-3196.

[18] F. Doyle, "An anti-windup input-output linearization scheme for SISO systems," Journal of Process Control, vol. 9, pp. 213-220, 1999.

[19] P. Lu, "Tracking control of nonlinear systems with bounded controls and control rates," Automatica, vol. 33, no. 6, pp. 1199-1202, 1997.

[20] N. Faiz, S. Agrawal, and R. Murray, "Differentially flat systems with inequality constraints: An approach to real-time feasible trajectory generation," Journal of Guidance, Control, and Dynamics, vol. 24, no. 2, pp. 219-227, 2001.

[21] P. Martin, R. Murray, and P. Rouchon, "Flat systems, equivalence, and trajectory generation," Caltech Technical Report, California Institute of Technology, Pasadena, CA, Tech. Rep., 2003.

[22] W. Liao, M. Cannon, and B. Kouvaritakis, "Constrained MPC using feedback linearization for systems with unstable inverse dynamics," in Proceedings of the American Control Conference, Portland, OR, June 2005, pp. 846-851.

[23] M. Bacic, M. Cannon, and B. Kouvaritakis, "Invariant sets for feedback linearisation based on nonlinear predictive control," IEE Proceedings in Control Theory Applications, vol. 152, no. 3, pp. 259265, 2005.

[24] V. Nevistic and J. Primbs, "Model predictive control: Breaking through constraints," in Proceedings of the IEEE Conference on Decision and Control, December 1996, pp. 3932-3937.

[25] N. El-Farra and P. Cristofides, "Switching and feedback laws for control of constrained switched nonlinear systems," in Hybrid Systems: Computation and Control, ser. LNCS 2289, C. Tomlin and M. Greenstreet, Eds. Springer-Verlag, March 2002, pp. 164-178.

[26] Y. Lin and E. Sontag, "A universal formula for stabilization with bounded controls," Systems and Control Letters, vol. 16, pp. 393-397, 1991.

[27] M. S. Branicky, "Multiple Lyapunov functions and other tools for switched and hybrid systems," IEEE Transactions on Automatic Control, vol. 43, no. 4, pp. 475-482, 1998.

[28] T. Hu and Z. Lin, "Composite quadratic Lyapunov functions for constrained control systems," IEEE Transactions on Automatic Control, vol. 48, no. 3, pp. 440-450, 2003.

[29] A. Glattfelder and W. Schaufelberger, Control systems with input and output constraints. Springer, 2003.

[30] A. Stoorvogel and A. Saberi, "Editorial: The challenge of constraints," International Journal of Robust and Nonlinear Control, vol. 14, p. 1085,2004

[31] G. Grimm, A. Teel, and L. Zaccarian, "Robust linear anti-windup synthesis for recovery of unconstrained performance," International Journal of Robust and Nonlinear Control, vol. 14, pp. 1133-1168, 2004.

[32] M. Turner and I. Postelthwaite, "Multivariable override control for systems with output and state constraints," International Journal of Robust and Nonlinear Control, vol. 14, pp. 1105-1131, 2004.

[33] C. Tomlin, I. Mitchell, A. Bayen, and M. Oishi, "Computational techniques for the verification of hybrid systems," Proceedings of the IEEE, vol. 91, no. 7, pp. 986-1001, 2003.

[34] I. Mitchell and C. Tomlin, "Level set methods for computation in hybrid systems," in Hybrid Systems: Computation and Control, ser. LNCS 1790, B. Krogh and N. Lynch, Eds. Springer Verlag, March 2000.

[35] P. Cardaliaguet, M. Quincampoix, and P. Saint-Pierre, “Optimal times for constrained non-linear control problems without local controllability," Applied Mathematics and Optimisation, vol. 35, pp. 1-22, 1997. 